\title{
Gaussian mixture models based 2D-3D registration of bone shapes for orthopedic surgery planning
}

\author{
Marta Valenti $^{1}$ - Giancarlo Ferrigno ${ }^{1}$ Dario Martina ${ }^{1} \cdot$ Weimin $\mathbf{Y u}^{2}$. \\ Guoyan Zheng ${ }^{2}$ Mohsen Akbari Shandiz ${ }^{3}$ Carolyn Anglin ${ }^{3} \cdot$ Elena De Momi $^{1}$
}

Received: 1 September 2015 / Accepted: 29 January 2016 / Published online: 23 March 2016

(C) International Federation for Medical and Biological Engineering 2016

\begin{abstract}
In orthopedic surgery, precise kinematics assessment helps the diagnosis and the planning of the intervention. The correct placement of the prosthetic component in the case of knee replacement is necessary to ensure a correct load distribution and to avoid revision of the implant. 3D reconstruction of the knee kinematics under weight-bearing conditions becomes fundamental to understand existing in vivo loads and improve the joint motion tracking. Existing methods rely on the semiautomatic positioning of a shape previously segmented from a $\mathrm{CT}$ or MRI on a sequence of fluoroscopic images acquired during knee flexion. We propose a method based on statistical shape models (SSM) automatically superimposed on a sequence of fluoroscopic datasets. Our method is based on Gaussian mixture models, and the core of the algorithm is the maximization of the likelihood of the association between the projected silhouette and the extracted contour from the fluoroscopy image. We evaluated the algorithm using digitally reconstructed radiographies of both healthy and diseased subjects, with a CT-extracted shape and a SSM as the 3D model. In vivo tests were done with fluoroscopically acquired images and subject-specific CT shapes. The results obtained are in line with the literature, but the computational time is substantially reduced.
\end{abstract}

Marta Valenti

marta.valenti@polimi.it

1 Department of Electronics, Information and Bioengineering, Politecnico di Milano, via Colombo 40, 20133 Milan, Italy

2 Universität Bern, Staffaucherstr. 78, 3014 Bern, CH Switzerland

3 University of Calgary, 2500 University Drive NW, Calgary, AB T2N 1N4, Canada
Keywords Statistical shape models · Gaussian mixture models $\cdot 2 \mathrm{D} / 3 \mathrm{D}$ registration $\cdot$ Orthopedic surgery $\cdot$ Image processing

\section{Introduction}

Osteoarthritis is a highly debilitating pathology that affects knees primarily of older people. With this inflammation, the cartilage degenerates, the distance between bones is reduced, and hypertrophy of the bones can occur, with the creation of osteophytes [36]. The Dutch Institute for Public Health estimates that worldwide almost $16 \%$ of men and $31 \%$ of women aged over 55 years have radiographic knee osteoarthritis [25]. In $12 \%$ of cases, the pathology reduces the motion of the knee and causes pain to the patient, often requiring the use of ambulatory aids [11]. The most used treatment for severe osteoarthritis is surgery. However, almost the $20 \%$ of the patients who undergo total knee arthroplasty (TKA) are not satisfied with their operation [2].

A preoperative study of knee kinematic under weightbearing conditions can improve the outcome of the surgery [32]. The representation of the joint kinematics in 3D space allows for understanding pain zones and ligament tensions, determining the best implant positioning and thus correcting nonalignments of the bone segments $[5,8,9,16,31$, $32,39]$. The acquisition of preoperative computer tomography $(\mathrm{CT})$ or magnetic resonance imaging (MRI) images gives a deep insight into the morphology of the structures, but these procedures are currently limited to static positioning and have high costs and radiation doses given to the patient. Mono and multi-plane fluoroscopies are the most accurate and used procedures to measure in vivo noninvasive kinematics of the knee $[32,39]$. The current clinical technique is mainly based on the operator's ability to 
correctly position the shape on the images [2]. This technique is time-expensive and error-prone, as it is based on human ability. Regardless of the number of fluoroscopic projections, two main automatic methods have been implemented to recover the correct pose of the bones in 3D space: intensity-based and feature-based methods.

Intensity-based methods need a preoperative CT scan in order to acquire the density of the patient's bone. The 2D-3D matching is then achieved comparing the digitally reconstructed radiograph (DRR) to the acquired fluoroscopic image and adjusting the rotation and translation parameters in order to minimize the differences between the two images in terms of pixel intensities [3, 18, 32]. Although very accurate, these methods require a previous $\mathrm{CT}$ to determine the bone density for the DRR creation and are computationally expensive for the number of pixel intensity comparisons that must be made to find the correct pose.

Feature-based methods are based on the contours of the bone shape that can be extracted from the fluoroscopic images using edge detector filters, such as Canny or Sobel $[12,22,23]$. These methods project the silhouette of the bone shapes and match it with the extracted edges. Usually, feature-based methods necessitate a previous morphological 3D dataset of the bone shapes, such as MRI or CT $[1,2,30]$. Recently, some authors have addressed the problem of needing the morphological scan and substituting it with a statistical shape model (SSM), a collection of shapes coming from atlas that can be deformed in order to represent accurately the target shape [5, 24, 34, 38]. In this way, costs are reduced and the patient is exposed to a lower radiation dose. However, computational time becomes an issue, as the whole sequence of poses takes some hours to be computed [7].

Our objective was to develop a new feature-based algorithm based on Gaussian mixture models (GMMs) that is able to register a 3D point set on a single or biplane image of the same object. We tested the algorithm with a SSM of the femur using a set of fluoroscopic images of the knee.
The goal was twofold: We addressed the problem of accurately recovering the pose of the knee in 3D space in a completely automatic way and also of ensuring the correct parameters for the deformation of the shape. In [21, 37], the authors addressed the problem of the registration of two point sets with a GMM fitting. The solution is given through an expectation conditional maximization (ECM) procedure that simplifies the original expectation maximization (EM) algorithm by Dempster et al. [17]. Both of these frameworks, however, assume working with point sets lying in the same (2D or 3D) space.

The proposed algorithm extends the state of the art to registering a 3D shape of a femur on a set of fluoroscopic images acquired during flexion-extension of the knee. It faces also the possibility to remove one of the two fluoroscopes in order to reduce the radiation dose and allow greater mobility in the fluoroscopic cone.

\section{Materials and methods}

The knee is imaged during a sequence of flexion-extension movements. A fluoroscopic tube returns a set of X-ray images. The source and the image plane are calibrated, and their pose in the world reference frame is known. The reconstruction of the femur kinematics is done through a series of features projection and backprojection from the fluoroscopic image plane to the 3D space. A 3D shape of the bone is used as a model to reconstruct the correct pose (Fig. 1).

\subsection{Datasets}

\subsubsection{D shape}

The 3D shape is defined as a set of points $\left(\mathbf{X}_{s}, s=1, \ldots, S\right)$ and triangles that can be either derived from the segmentation of a volumetric image dataset (CT or MRI) [26] or a SSM
Fig. 1 X-ray source ${ }^{j} S$ projects rays on the image plane. The image is processed, and the contours ${ }^{j} y_{n}$ are extracted using a Canny edge detector (black points on the image). In the middle, the shape is formed by points $X_{s}$ and triangles. The silhouette of the model, $X_{m}$ in red, is made up of those points that share a contour edge, i.e., an edge shared by two triangles with normals (arrows) pointing in different directions from the source. The zoom on the right shows the normals of the triangles that point in different directions

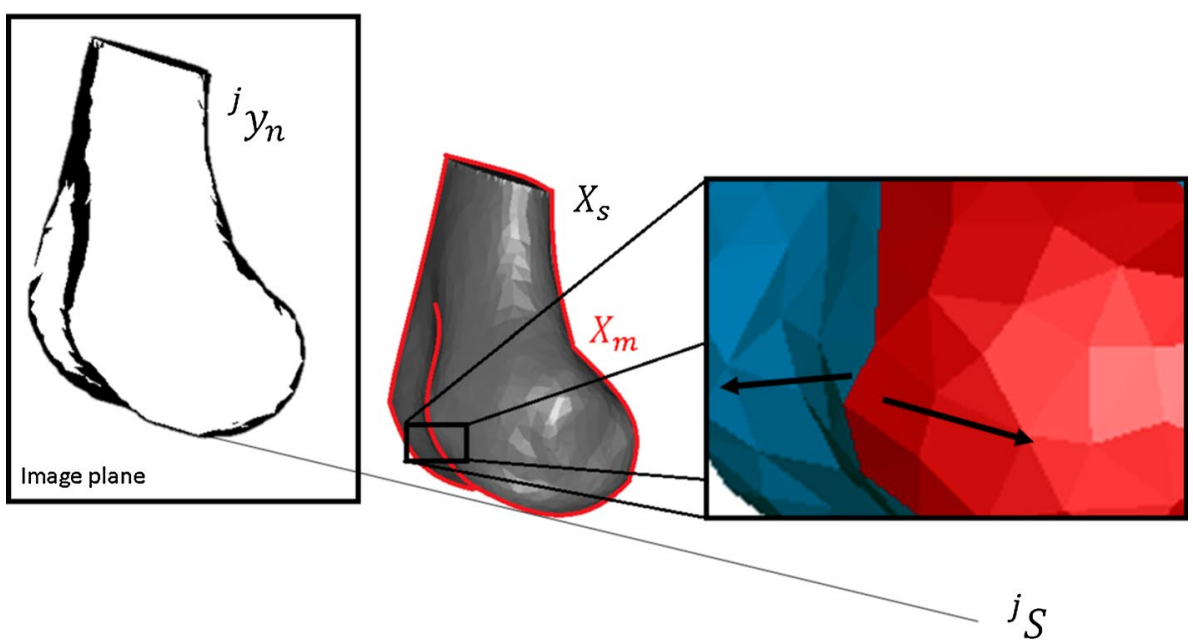


[14]. We define the patient-specific 3D shape as $3 \mathrm{D} S_{\mathrm{CT} / \mathrm{MRI}}$ and the SSM as $3 \mathrm{D} S_{\mathrm{SSM}}(\mathrm{A})$. A Gaussian distribution $\left(\mathbf{X}_{s}, \Sigma_{s}\right)$ is associated with each $3 \mathrm{D}$ point of the model. The isotropic covariance can be expressed as $\Sigma_{s}=\sigma_{s} \mathbf{I}_{3}$.

The silhouette of the model is made up of those points that share a contour edge, i.e., an edge shared by two facets with normals pointing in different directions from the source. The silhouette points are then a subset of the shape points $\mathbf{X}_{m}, m=1, \ldots, M<S$. The model silhouette is projected on the image plane $\mathbf{x}_{m}, m=1, \ldots, M$.

\subsubsection{D contours}

The segmentation of the contour of the shape on the images is performed using a semiautomatic algorithm based on gradient enhancement of the image and a spline interpolation between user-picked points [34]. The selected pixels, belonging to the segmentation, can be transformed in 3D points using the known image calibration parameters $\left(\mathbf{y}_{n}, n=1, \ldots, N\right)$.

\subsection{Registration}

\subsubsection{Initialization}

A manual initialization is necessary to define the initial pose for the shape. Seven landmark points $L_{i}, i=1, \ldots, 7$ are identified on the 3D shape 3DS. The user is asked to select the same points on the images [33]. The backprojected lines from the user-selected points to the corresponding source identify seven landmarks in the 3D space. Using corresponding point registration [4], we find the homogeneous transformation matrix $\mathbf{T}$ that maps the shape in the calibrated image space. The accuracy in finding the exact points on the images and the resulting initialization matrix $\mathbf{T}$ is not crucial, as the whole algorithm has been proven to be robust against initialization: we checked the robustness of the method initializing the algorithm with different poses, starting from the correct pose and gradually adding up to 5 $\mathrm{cm}$ and $5^{\circ}$ of uniform random noise to the correct matrix.

\subsubsection{Expectation conditional maximization}

The expectation step (E-step) involves the computation of the posterior probability $p_{m n}$ that every point of the projected shape on the image plane $\left(\mathbf{x}_{m}, m=1, \ldots, M\right)$ is associated with a point of the contour $\left(\mathbf{y}_{n}, n=1, \ldots, N\right)$ :

$p_{m n}=\mathcal{P}\left(Z: \mathbf{y}_{n} \rightarrow \mathbf{x}_{m} \mid \mathbf{y}_{n} ; \theta, \sigma\right)$

where $Z$ is the association operator between a projected point $\mathbf{x}_{m}$ of the shape's silhouette $\mathbf{X}_{m}$ with a point of the contour $\mathbf{y}_{n}$ extracted from the image (B). The set of unknown variables is composed by the registration parameters $\theta[6 \times 1]=\left\{q_{1}, q_{2}, q_{3}, t_{1}, t_{2}, t_{3}\right\}$ where $q_{i}, i=1,2,3$ are the Euler angles and $t_{i}, i=1,2,3$ are the translation values, and the set of $3 \mathrm{D}$ points variances $\sigma_{1}^{2}, \ldots, \sigma_{M}^{2}$ :

$\psi=\left(\theta, \sigma_{1}^{2}, \ldots, \sigma_{M}^{2}\right)$

As the direct maximization of the likelihood is intractable, the minimization of the negative log-likelihood $(E(\psi))$ will instead be taken as the objective [17]:

$E(\psi)=-\sum_{n=1}^{N} \log \sum_{m=1}^{M} \mathcal{P}\left(\mathbf{X}_{m}\right) \mathcal{P}\left(\mathbf{y}_{n} \mid \mathbf{X}_{m}\left(\theta ; \sigma_{m}^{2}\right)\right)$

The minimization function can thus be transformed in:

$$
\begin{aligned}
E(\psi)= & \frac{1}{2} \sum_{n=1}^{N} \sum_{m=1}^{M} \frac{p_{n m}}{\sigma_{m}}\left[\left(\left\|\mathbf{y}_{n}-\mathbf{X}_{m}(\theta)\right\|^{2}\right)\right. \\
& \left.+3 \sigma_{m}^{2} \log \left(\sigma_{m}^{2}\right)\right]+\frac{\rho}{2}\|L(\phi)\|^{2}
\end{aligned}
$$

where $\|L(\phi)\|^{2}$ is a regularization parameter over the transformation, and $\rho$ weights its contribution to the minimization.

The ECM algorithm allows a simplification of the problem dividing the minimization in two steps (CM-step):

- minimization of Eq. (3) over the registration parameters - update of the variances using the newly estimated registration parameters

In the case of a rigid transformation, the parameter $\|L(\phi)\|^{2}$ is equal to 0 , and the solution of the problem is given by a least-squares fitting of two 3D point sets, as described in [4].

If the shape to be registered is a $3 \mathrm{D} S_{S S M}$, the parameters to be estimated are the shape coefficient vectors $\beta_{k}$, and the regularization term assumes the form of the Mahalanobis distance, with $\lambda_{k}^{2}$ eigenvalues of the SSM:

$\|L(\phi)\|^{2}=\sum_{k=1}^{K^{\prime}} \frac{\beta_{k}^{2}}{\lambda_{k}^{2}}$

All the shape coefficients are determined with a closedform solution as described in [37].

The algorithm converges to a minimum if the percentage difference between the likelihood of two consecutive frames is below a predefined threshold. Specifically:

$\frac{\mathcal{L}^{(t)}(\mathcal{X} \mid \mathcal{Y})-\mathcal{L}^{(t-1)}(\mathcal{X} \mid \mathcal{Y})}{\mathcal{L}^{(t-1)}(\mathcal{X} \mid \mathcal{Y})}<\epsilon$

where $\mathcal{X}$ and $\mathcal{Y}$ are, respectively, the GMM and the observations. The likelihood of the current step is defined as: 
Table 1 For each subject, the age, gender, and osteoarthritic grade are indicated

\begin{tabular}{lllllll}
\hline Subject & Age & Gender & Osteoarthritic grade & CT & DRR $_{0 / 10 / 90}$ & Fluoroscopies $_{0 / 10}$ \\
\hline$S_{0}$ & 47 & F & None & x & $0^{\circ}: 3^{\circ}: 72^{\circ}$ & \\
$S_{1}$ & 67 & M & Severe & x & $0^{\circ}: 8^{\circ}: 80^{\circ}$ & $0^{\circ}: 15^{\circ}: 90^{\circ}$ \\
$S_{2}$ & 75 & M & Mild & x & $0^{\circ}: 8^{\circ}: 80^{\circ}$ & $0^{\circ}: 15^{\circ}: 90^{\circ}$ \\
$S_{3}$ & 82 & F & Moderate & x & $0^{\circ}: 8^{\circ}: 80^{\circ}$ & $0^{\circ}: 15^{\circ}: 90^{\circ}$ \\
$S_{4}$ & 65 & F & Mild & x & & $0^{\circ}: 15^{\circ}: 90^{\circ}$ \\
$S_{5}$ & 75 & M & Severe & x & & $0^{\circ}: 15^{\circ}: 90^{\circ}$ \\
$S_{6}$ & 71 & F & Moderate & x & & $0^{\circ}: 15^{\circ}: 90^{\circ}$ \\
$S_{7}$ & 82 & M & Mild & x & & $0^{\circ}: 15^{\circ}: 90^{\circ}$ \\
\hline
\end{tabular}

All the femurs analyzed were right femurs. The $\mathrm{DRR}_{0 / 10 / 90}$ values indicate the angles for which we generated the DRRs. We specify the starting and ending angle, with the step used. The Fluoroscopies ${ }_{0 / 10}$ values indicate the angles of the fluoroscopic images
$\mathcal{L}(\mathcal{X} \mid \mathcal{Y})=\prod_{n=1}^{N} \sum_{m=1}^{M} \alpha_{m} \cdot\left(\sigma_{m}^{-2} e^{\frac{-\left\|\mathbf{y}_{n}-\mathbf{x}_{m}\right\|^{2}}{2 \sigma_{m}^{2}}}\right)$

In (7), $\alpha_{m}$ is the component prior of the specific model [15].

\subsection{Validation protocol}

For this study, we used a single SSM dataset with 69 shapes. All the patients were imaged using MRI scans and manually segmented in order to find the $3 \mathrm{D}$ shape. The datasets included both male and female bones, with either right or left mirrored knee shapes. Those shapes were used to define the SSM as described in A [33].

The subject dataset is the following (Table 1):

- one healthy subject $S_{0}$ who underwent a CT scan (Sensation Cardiac 64, Siemens). The CT dataset is composed of 59 slices of $512 \times 512$ pixels each $(0.7890625$ $\mathrm{mm} /$ pixel). The slice thickness is $2 \mathrm{~mm}$, and the space between slices is $1.7 \mathrm{~mm}$. The CT dataset was used to generate the DRR for evaluation purposes.

- Seven osteoarthritic patients $\left(S_{i}, i=1, \ldots, 7\right)$ eligible for TKA with different grades of osteoarthritis. The

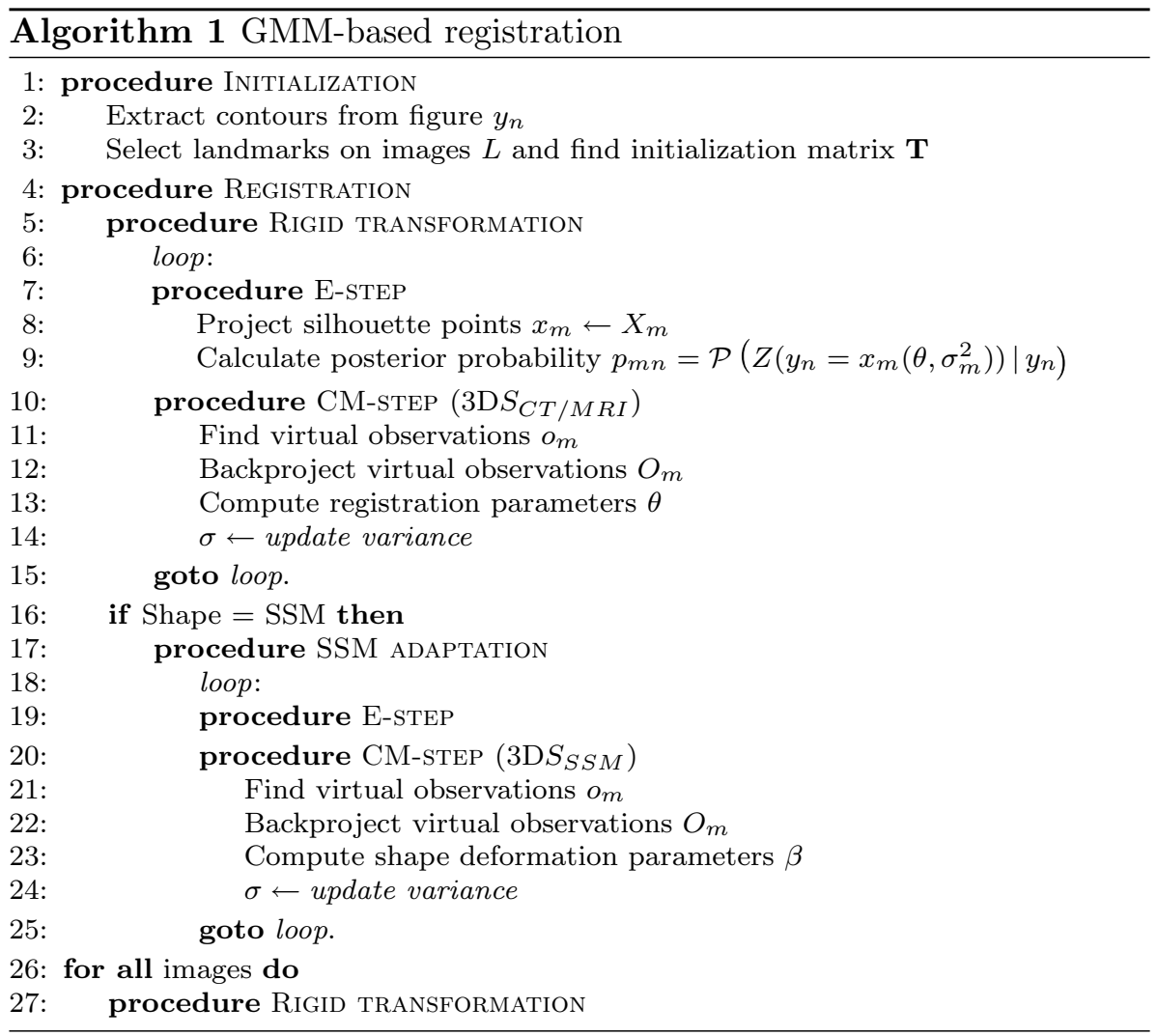




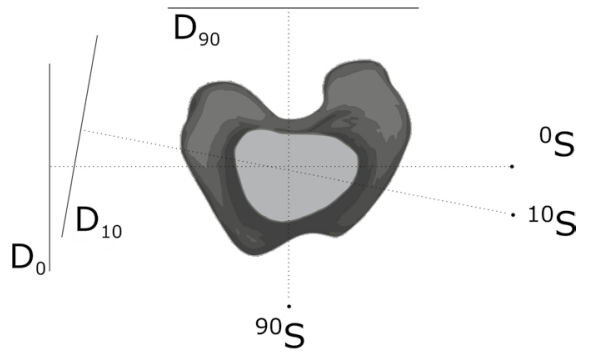

(a) DRR creation

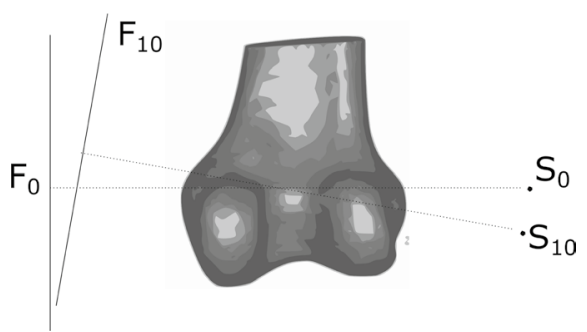

(b) Fluoroscopy acquisition
Fig. 2 Description of the virtual environment setup for the DRR creation and fluoroscopic acquisitions. The source $S_{0}$ was established on the medio-lateral axis, as well as the center of $D_{0}$ and $F_{0}$. For the DRR, $D_{10}$ is obtained rotating the source-plane axis of $10^{\circ}$ on the

patients underwent a preoperative $\mathrm{CT}$ scan from which the $3 \mathrm{D} S_{C T}$ of the femur was segmented [20]. The CT datasets were composed of DICOM images acquired with a SIEMENS Sensation 64 CT machine. Each slice is $512 \times 512$ pixel $(0.3516 \mathrm{~mm} / \mathrm{pixel})$ with a slice thickness of $0.6 \mathrm{~mm}$ and a spacing between slices of $0.4 \mathrm{~mm}$. All the patients were also imaged at seven fixed flexion angles using two sequential fluoroscopic projections with an AXIOM Luminos dRF flatbed (Siemens; Berlin, Germany) [2]. The first image was taken with the projector placed horizontally (lateral image), while the second was taken with the source at $10^{\circ}$ below horizontal. Both projections were calibrated using custommade calibration software [29]. The fixed flexion angles $\left(0^{\circ}, 15^{\circ}, 30^{\circ}, 45^{\circ}, 60^{\circ}, 75^{\circ}, 90^{\circ}\right)$ were obtained using steps of different customized heights on which the subject could step up. Three subjects $\left(S_{1}, S_{2}, S_{3}\right)$ with different grades of osteoarthritis were selected to generate the DRRs of diseased knees.

All the patients signed an informed consent, and the institutional review board approved the study.

In order to assess the model reconstruction performances, all the CT datasets were segmented using Amira ${ }^{\circledR}$ (VSG-FEI, France), and the anatomical reference frame was defined as in [28].

The DRR is built integrating the density of each voxel of the CT along the direction of each ray as in [27]. Three different sources and image planes were simulated for each patient, resulting in three sets for each patient: the first image shows a lateral view of the femur $\left(L_{0}\right)$, the second and third images show a view rotated on the sagittal plane of $10\left(L_{10}\right)$ and $90^{\circ}\left(L_{90}\right)$ (see Fig. 2) [35]. For subject $S_{0}$, we rotated the femur from $0^{\circ}$ to $72^{\circ}$ with a step of $3^{\circ}$ generating the ground truth pose $\left(\mathbf{T}_{G T_{0}}^{i}, i=0^{\circ}, 3^{\circ}, \ldots, 72^{\circ}\right)$. The three pathological DRRs were instead created rotating the horizontal plane. $D_{90}$ is obtained rotating the source-plane axis of $90^{\circ}$, having it correspondent to the anteroposterior axis. For the fluoroscopies, $F_{10}$ is obtained rotating the source-plane axis of $10^{\circ}$ on the medio-lateral axis a DRR creation. b Fluoroscopy acquisition

femur from $0^{\circ}$ to $80^{\circ}$ with a step of $8^{\circ}$ generating the ground truth pose $\left(\mathbf{T}_{G T_{1,2,3}}^{i}, i=0^{\circ}, 8^{\circ}, \ldots, 80^{\circ}\right)[34]$.

\subsubsection{Tests}

The initialization is done as described in paragraph 2.2.1. The tracking consists in finding the correct pose of the 3DS for all the images provided for a given flexion sequence. The tests were performed with six different conditions $C_{1, \ldots, 7}$ :

$C_{1}: \quad 3 \mathrm{DS}$ pose initialization with $D_{0}\left(0^{\circ}\right)$ and $D_{90}\left(0^{\circ}\right)$ and tracking with $D_{0}$ and $D_{90}$ for all angles with $S_{0}, \ldots, S_{3}$

$C_{2}: \quad 3 \mathrm{DS}$ pose initialization with $D_{0}\left(0^{\circ}\right)$ and $D_{90}\left(0^{\circ}\right)$ and tracking with $D_{0}$ for all angles with $S_{0}, \ldots, S_{3}$

$C_{3}$ : $3 \mathrm{DS}$ pose initialization with $D_{0}\left(0^{\circ}\right)$ and $D_{10}\left(0^{\circ}\right)$ and tracking with $D_{0}$ and $D_{10}$ for all angles with $S_{0}, \ldots, S_{3}$

$C_{4}: \quad 3 \mathrm{DS}$ pose initialization with $D_{0}\left(0^{\circ}\right)$ and $D_{10}\left(0^{\circ}\right)$ and tracking with $D_{0}$ for all angles with $S_{0}, \ldots, S_{3}$

$C_{5}$ : $\quad 3 \mathrm{D} S$ pose initialization with $F_{0}\left(0^{\circ}\right)$ and $F_{10}\left(0^{\circ}\right)$ and tracking with $F_{0}$ and $F_{10}$ for all angles with $S_{1}, \ldots, S_{7}$

$C_{6}: \quad 3 \mathrm{D} S$ pose initialization with $F_{0}\left(0^{\circ}\right)$ and $F_{10}\left(0^{\circ}\right)$ and tracking with $F_{0}$ for all angles with $S_{1}, \ldots, S_{7}$

For each trial, both the SSM (3DSSSM) and the CT segmented shape $\left(3 \mathrm{D} S_{\mathrm{CT}}\right)$ were used. In this way, both the pose and the shape estimation can be evaluated with respect to the ground truth. The trials $\mathrm{C} 1-\mathrm{C} 4$, performed using artificially constructed DRR, highlight the pose errors between the registered model and the source CT, from which the images were created. When the ( $\left.3 \mathrm{D} S_{\mathrm{SSM}}\right)$ is used, the shape reconstruction is compared to the known patient-specific shape, in order to check the similarity between the two poses. 


\subsubsection{Results evaluation}

The homogeneous matrix $\mathbf{T}_{\theta}^{i}$ was constructed from the optimal parameters $\theta$ returned by the ECM algorithm. In case of conditions $C_{1}, \ldots, C_{4}$, we computed $\mathbf{T}_{\text {residual }}^{i}=\mathbf{T}_{G T}^{i}{ }^{-1} \cdot \mathbf{T}_{\theta}^{i}$. The errors were presented as rotations (in terms of Euler angles) and translations of $\mathbf{T}_{\text {residual }}^{i}$.

For the conditions $C_{3}, \ldots, C_{6}$, edge-to-surface (E2S) distance was computed [7]. E2S is defined as the Euclidean distance between a point on the 3D shape and the closest point on the associated contour pixel backprojection. In this way, E2S does not require the ground truth pose to evaluate the accuracy of the algorithm. Kruskal-Wallis test with $p<0.05$ was used to asses if the results for $C_{3}, \ldots, C_{6}$ using $3 \mathrm{D} S_{C T}$ and $3 \mathrm{D} S_{\mathrm{SSM}}$ were statistically different.

\section{Results}

Concerning the initialization validation, the method proved to be robust under different conditions, with a maximum allowed error of $2 \mathrm{~cm}$. The results showed that with an error higher than $2 \mathrm{~cm}$ and $2^{\circ}$, the system is not assured to converge, while with a lower error the system converges with final errors lower than $1 \mathrm{~mm}$. The initialization can thus be considered independent from the operators' skills.

Figure 3 represents the results of an optimization of a $3 \mathrm{D} S_{C T}$ in $C_{1}$ condition. As shown in the figure, the points of the projected silhouette tend to overlay the points of the contour, in order to minimize the distance between the two datasets.

In order to better understand the results, the errors have been expressed in the anatomical axes of the subject.

Figure 4 shows the values of rotation and translation of $\mathbf{T}_{\text {residual }}$ in the case of the healthy subject $S_{0}$. As can be seen, in case of single-plane tracking or dual-plane tracking with $D_{0}$ and $D_{10}$, the error in the medio-lateral axis is increasing up to $3 \mathrm{~cm}$ with the flexion angle. The translation errors on the other axis, as well as the rotation errors on every axis, are bounded between some $\mathrm{mm}$ and $1 \mathrm{~cm}$ in the case of $3 \mathrm{D} S_{\mathrm{SSM}}$. The results are more accurate using the $3 \mathrm{D} S_{C T}$. In this case, the errors are below $1 \mathrm{~cm}$ also in the out-of-plane axis (medio-lateral).

Figure 5 shows the errors in terms of rotation and translation for conditions $C_{1}, \ldots, C_{4}$ and using $3 \mathrm{D} S_{\mathrm{CT}}$ and $3 \mathrm{D} S_{\mathrm{SSM}}$. In these figures, it can be seen that the error is generally lower in the case of $3 \mathrm{D} S_{\mathrm{CT}}$, apart from the mediolateral axis of $S_{2}$ when the error raises up to $6 \mathrm{~cm}$. In $S_{3}$, an angle-dependent trend is clearly visible, similar to those shown in Fig. 4.

The edge-to-surface index is represented in Fig. 6 for the only tests with $L_{0}$ and $L_{10}$ images and for subject $S_{1}, S_{2}, S_{3}$. Results are presented as a population of E2S root mean

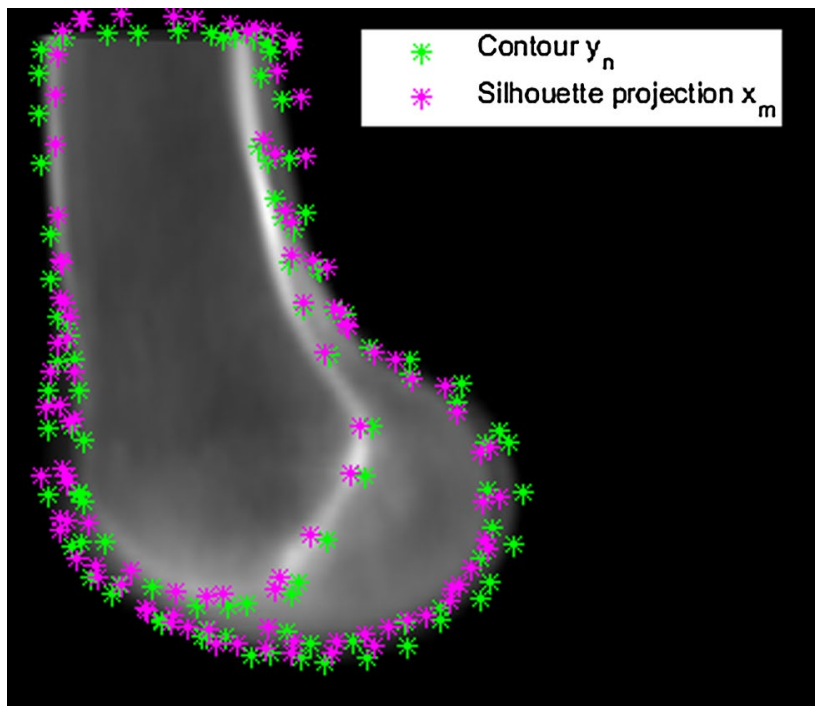

Fig. 3 Representation of the contour points and the silhouette points projected after the optimization of the algorithm

square (RMS) for each pose of the trials. Results were grouped for the type of images analyzed (DRR or fluoroscopies). The parentheses above the boxplot indicate that Kruskal-Wallis test returned differences in the distribution median.

In Fig. 7 are shown the E2S results for the fluoroscopic acquired images. Patients $S_{1}, \ldots, S_{7}$ proved to be statistically different only in a few cases, with $S_{2}$ that has the highest differences. The lack of difference between different methods using the same subject indicates that no statistical difference is evidenced among the different options for the algorithm (CT or SSM shape and single or double image). The difference shown with $S_{2}$ can be ascribed to a very distal cut of the diaphysis, which compromises the ability of the system to recover the correct pose of the shape.

\section{Discussion}

This paper describes an innovative method to obtain the pose of the femur from single or biplane fluoroscopies to be used in knee tracking for accurate planning of orthopedic surgery, starting from a patient-specific model (obtained from volumetric dataset) or from a generic SSM. In the latter case, the radiation dose for the patient can be reduced. Knowing knee kinematics allows understanding pain zones associated with tensions of the ligaments and contact of the bones on each other, improving the outcome of the surgery.

Our registration method is based on GMMs and solves the maximum likelihood (ML) problem using an ECM approach, which allows significantly reducing the computational costs. Only a few seconds per image are enough to 

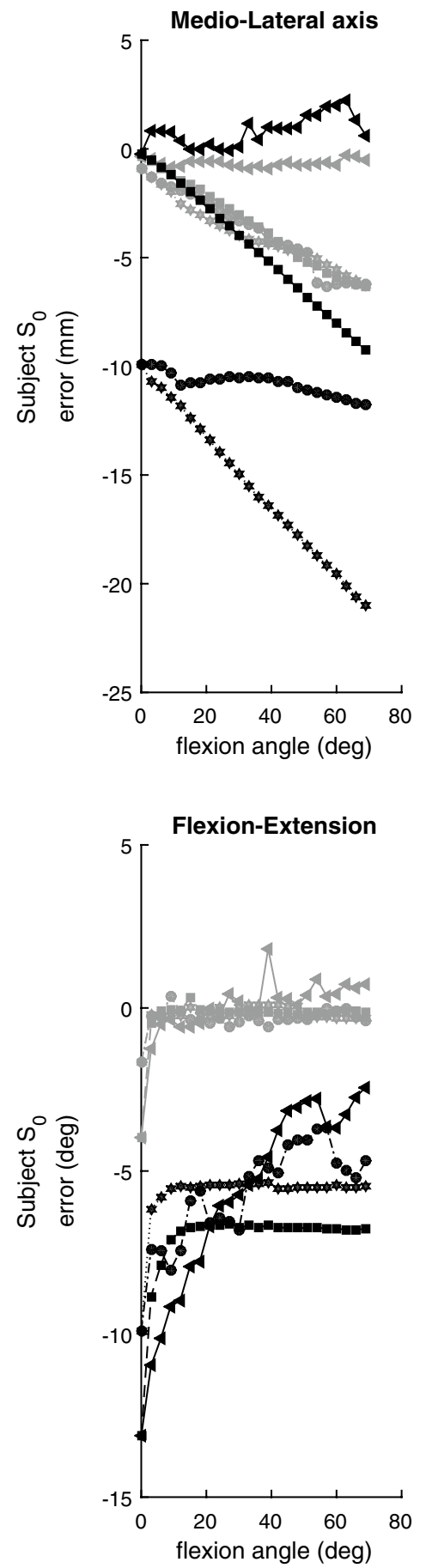
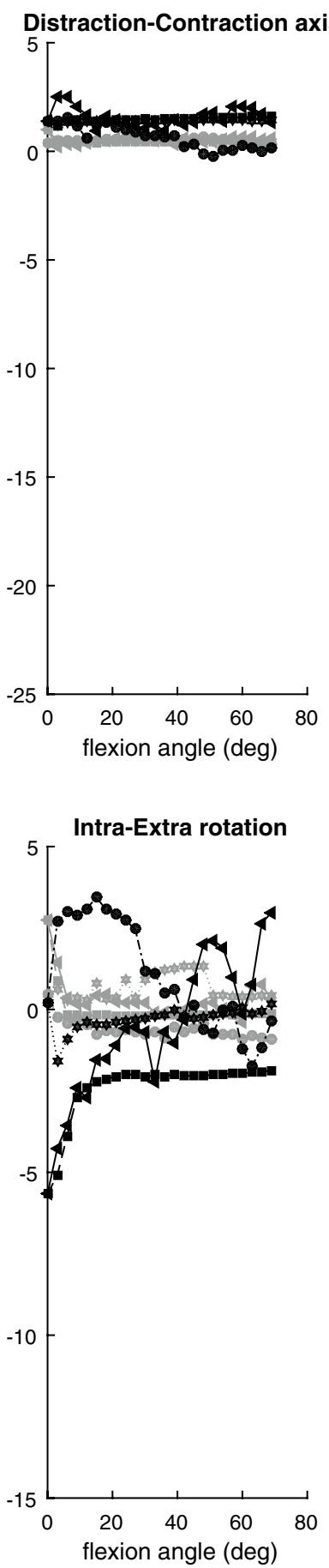
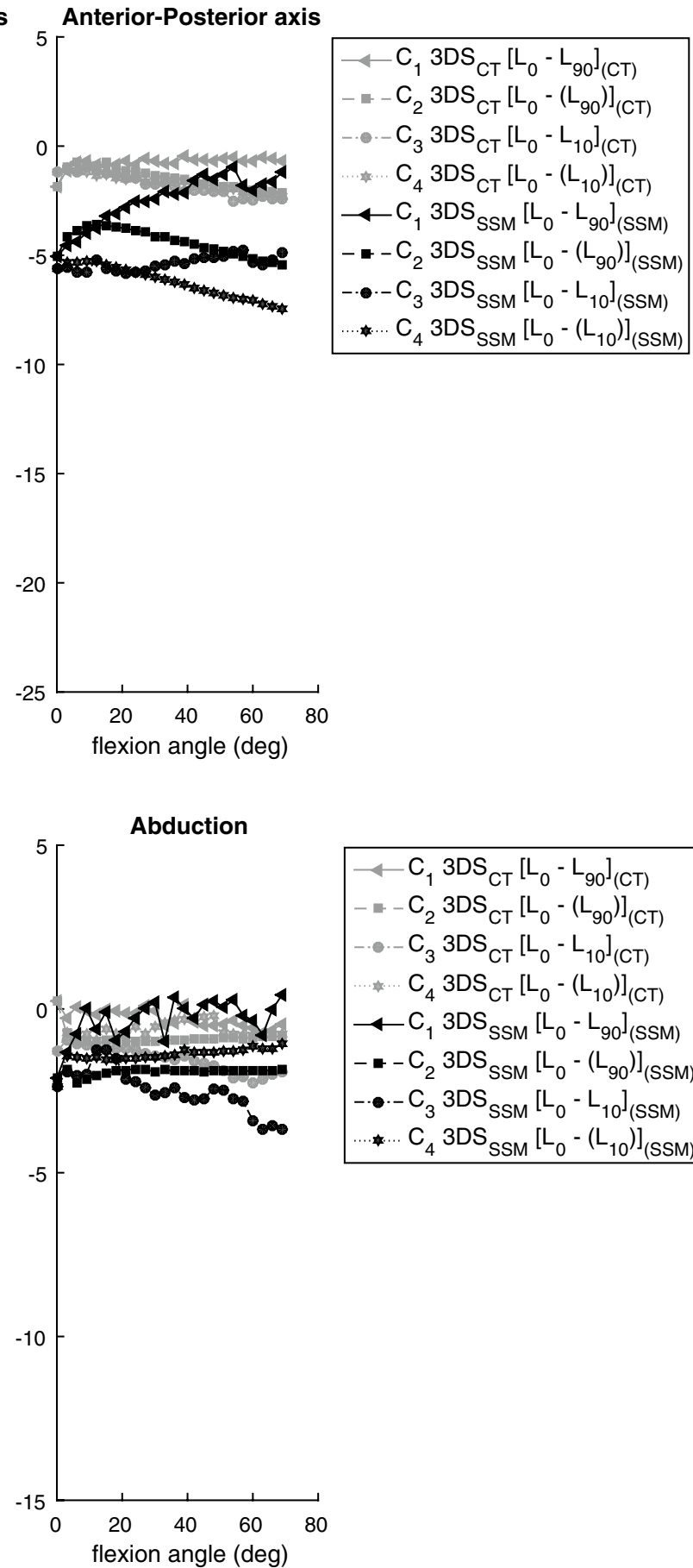

$$
\begin{array}{|l}
-\mathrm{C}_{1} 3 \mathrm{DS} \mathrm{C}_{\mathrm{CT}}\left[\mathrm{L}_{0}-\mathrm{L}_{90}\right]_{(\mathrm{CT})} \\
-\mathrm{C}_{2} 3 \mathrm{DS} \mathrm{CT}_{\mathrm{CT}}\left[\mathrm{L}_{0}-\left(\mathrm{L}_{90}\right)\right]_{(\mathrm{CT})} \\
\cdots \mathrm{C}_{3} 3 \mathrm{DS} \mathrm{C}_{\mathrm{CT}}\left[\mathrm{L}_{0}-\mathrm{L}_{10}\right]_{(\mathrm{CT})} \\
-\mathrm{C}_{4} 3 \mathrm{DS} \mathrm{S}_{\mathrm{CT}}\left[\mathrm{L}_{0}-\left(\mathrm{L}_{10}\right)\right]_{(\mathrm{CT})} \\
-\mathrm{C}_{1} 3 \mathrm{DS} \mathrm{S}_{\mathrm{SSM}}\left[\mathrm{L}_{0}-\mathrm{L}_{90}\right]_{(\mathrm{SSM})} \\
-\mathrm{C}_{2} 3 \mathrm{DS} \mathrm{SSM}_{\mathrm{SS}}\left[\mathrm{L}_{0}-\left(\mathrm{L}_{90}\right)\right]_{(\mathrm{SSM})} \\
\cdots \mathrm{C}_{3} 3 \mathrm{DS} \mathrm{SSM}_{\mathrm{SS}}\left[\mathrm{L}_{0}-\mathrm{L}_{10}\right]_{(\mathrm{SSM})} \\
\mathrm{C}_{4} 3 \mathrm{DS} \mathrm{SSM}_{\mathrm{SS}}\left[\mathrm{L}_{0}-\left(\mathrm{L}_{10}\right)\right]_{(\mathrm{SSM})}
\end{array}
$$

Fig. 4 Translation and rotation error of $\mathbf{T}_{\text {residual }}$ for $S_{0}$

ensure the convergence of the system to the correct result, while for the previous methods [6, 34], several hours were needed to reach convergence. Compared to previous works that used GMMs to register two different shapes on each other [13, 21, 37], our approach implements a 2D/3D registration, addressing the problem of a registration between two datasets with different dimensions. The method works in a semiautomatic way: it requires a rough initialization from the user and a threshold parameter to extract the contours with a Canny edge detector, saving time and augmenting the accuracy.

The results presented in our analysis show that the difference between the SSM and the CT-extracted shape is significant only in a few cases, especially with diseased 

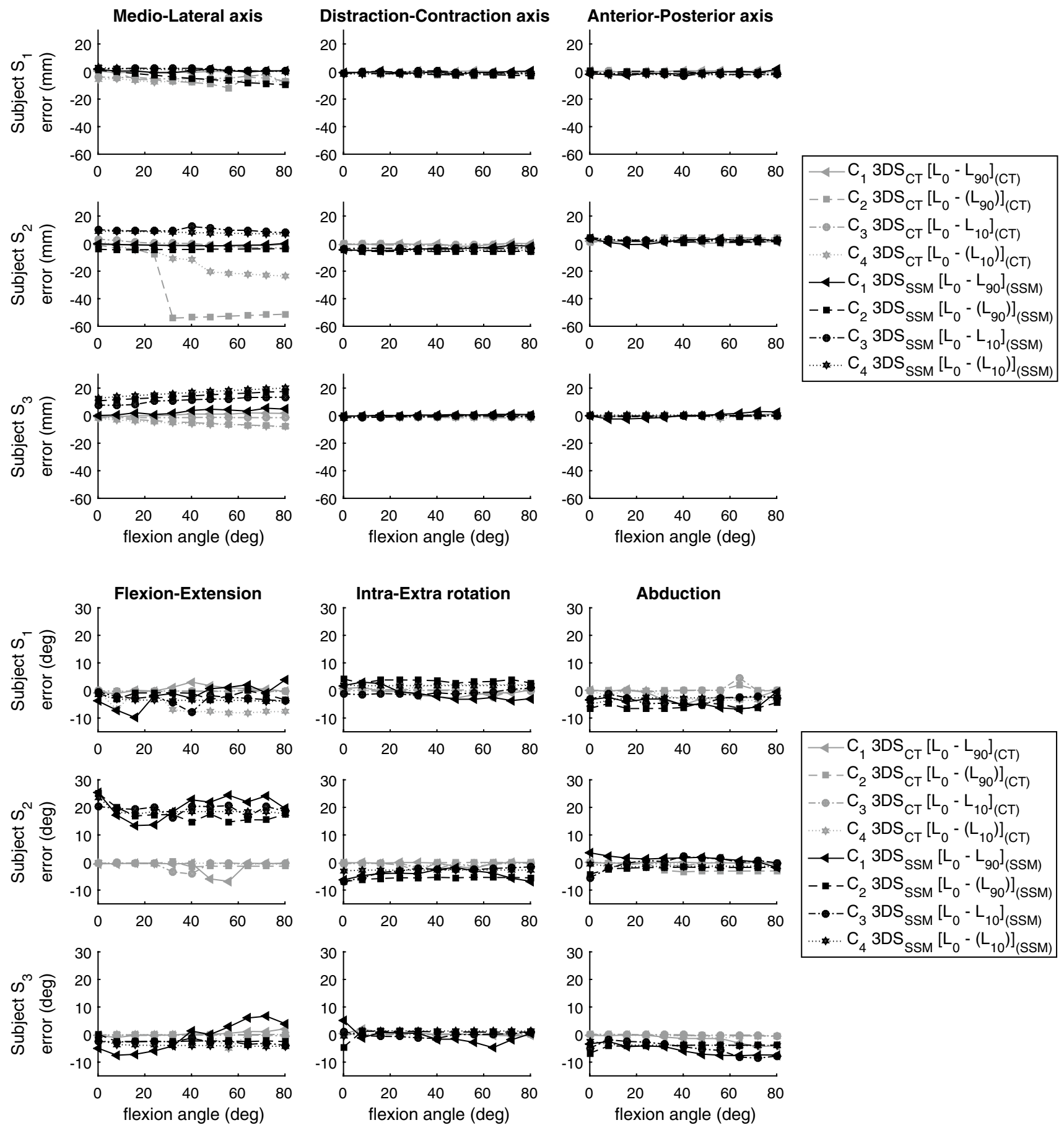

Fig. 5 Translation and rotation error of $\mathbf{T}_{\text {residual }}$ for $S_{1}, S_{2}, S_{3}$

shapes. This can be due to limited morphing capability of the statistical model given by the low number (69) of healthy knees used as datasets [33]. The small number of subjects used to build the SSM causes the low ability of the model to resemble the subject-specific shape even in the case of the healthy subject which shows bigger deformations and pose errors than expected.
Overall, the implemented registration method proved to have results comparable to the literature. In [7], the authors found a translation error of a few $\mathrm{mm}(0.48-0.81$ for the median accuracy, and approximately $2 \mathrm{~mm}$ for the precision) that are comparable with the results we found in our experiments in the case of DRR tracking with $S_{0}$ and $C_{1}$. The results presented in [24] show a similar trend, with a 
Fig. 6 Comparison of the edge-to-surface index for subjects $S_{1}, S_{2}, S_{3}$ with DRR or fluoroscopic images. The brackets above the box indicate statistical differences between the medians, according to Kruskal-Wallis test

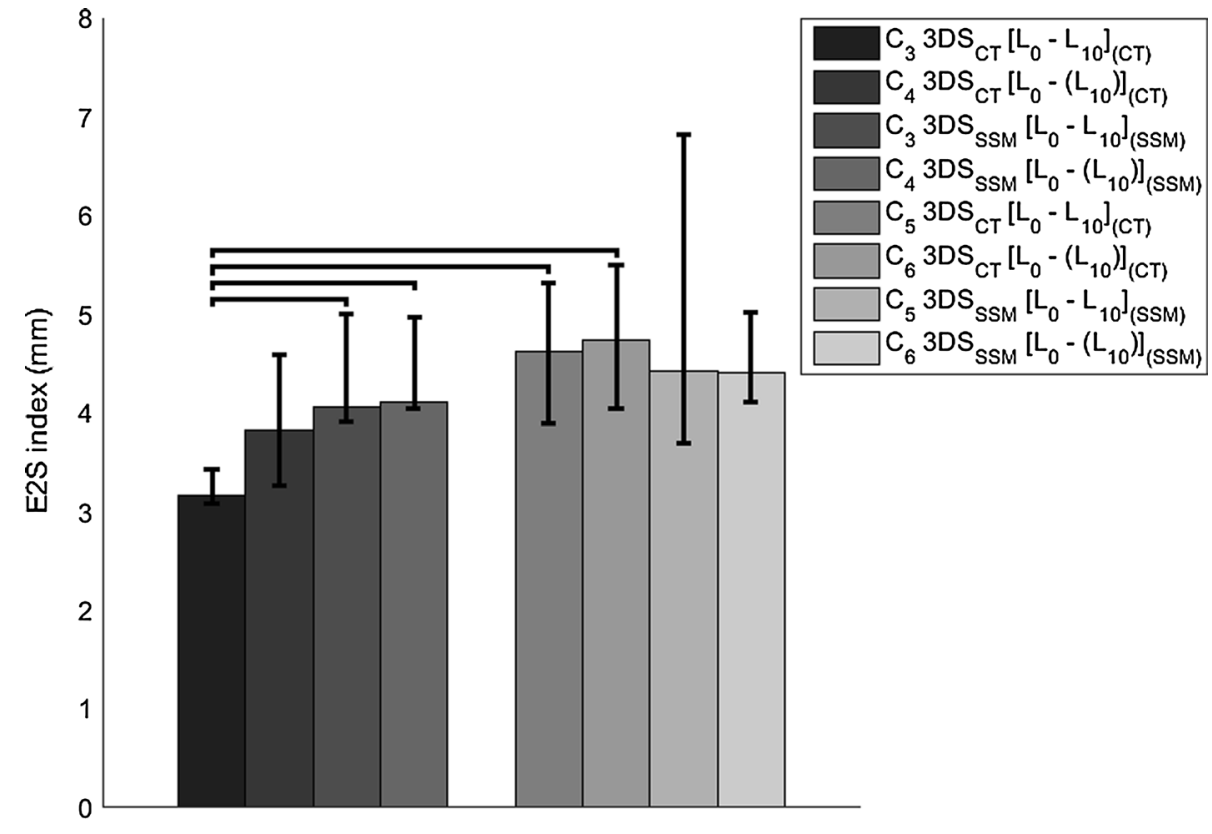

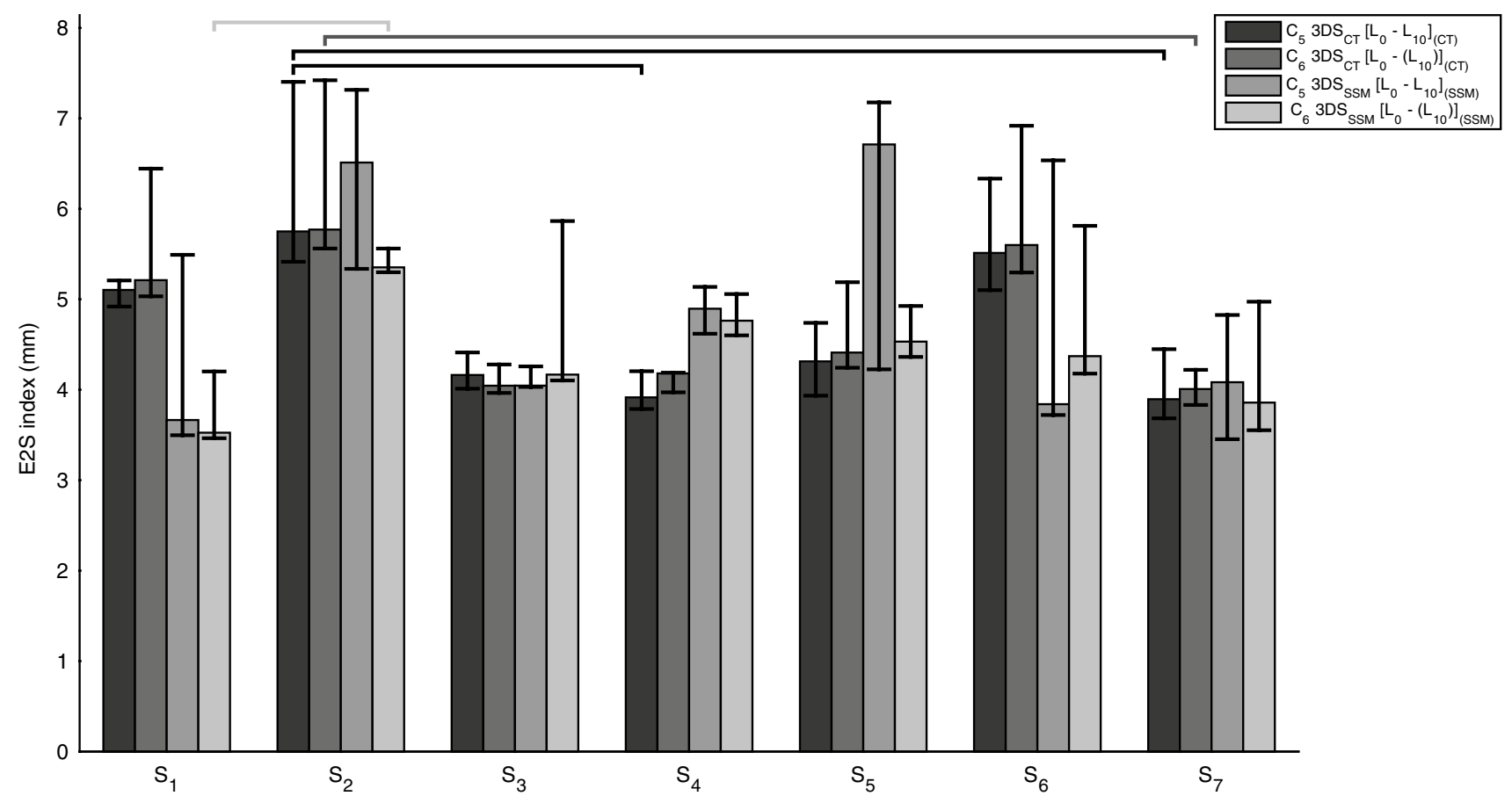

Fig. 7 Edge-to-surface index for each subject with fluoroscopic images. The brackets indicate significant difference between two groups

few $\mathrm{mm}$ of error (up to $4.3 \mathrm{~mm}$ ) and some degrees of inaccuracy (up to $4^{\circ}$ ).

The fluoroscopic image projection angle influences the tracking accuracy of the depth dimension. The ML axis has bigger errors compared to the others axis, because reducing the angle between the two projections from $90^{\circ}$ to $10^{\circ}$ decreases the pose determination accuracy [19]. The same behavior regarding out-of-plane errors can be observed when the tracking is performed with single-plane fluoroscopy. The error in depth increases from frame to frame, as there are no constraints on this axis. In the case of diseased patients, the error in depth can reach up to $6 \mathrm{~cm}$ (Fig. 5). In fact, the indetermination given by the single projection must be added to the nonperfect correspondence between 
the extracted contours and the statistical shape. The same considerations could be asserted relative to the rotation errors. Apart from subject $S_{2}$, which has a very distal cut of the diaphysis (due to a tight joint intraoperatively) that compromises the reconstruction of the correct pose, the results are in line with those presented in $[1,31]$, who found errors below $1 \mathrm{~cm}$ with a higher distance in the out-of-plane axis.

The evaluation of the accuracy with fluoroscopic images was performed using the E2S index to allow an evaluation of the accuracy without knowing the correct pose of the ground truth. The results show a statistical difference in the case of tracking with the $3 \mathrm{D} S_{\mathrm{CT}}$ (Fig. 6). This is probably due to a different Canny threshold, which influenced the correct positioning of the shape. The same error is not visible in the case of $3 \mathrm{D} S_{\mathrm{SSM}}$ thanks to the lower deformation of the shape. These results are comparable with the one stated by [7] who found an error of approximately $2 \mathrm{~mm}$. However, their dataset had a proximal cut of the diaphysis, augmenting the accuracy of the algorithm. Dealing with pathological subjects is more challenging, especially with a reduced set of shapes that created the SSM. Results are in the order of some mm (depending on the condition) and could be still acceptable to evaluate the kinematics of the knee. The method proved to be robust and efficient, especially when used with patient-specific shapes. Regarding the single-plane registration, Figs. 6 and 7 show that there is no statistical difference due to the difference in the number of image planes adopted. A single-plane registration allows the reduction in radiation dose given to the patient and enlarges the possible movements inside the field of view of the fluoroscope. Future work will increase the number of shapes to construct the SSM and include also the tibia in the study, in order to have the joint angle value for a proper kinematic analysis. For single-image tracking, higher constraints on the depth axis must be implemented (e.g., a constrained motion of max $1 \mathrm{~mm}$ between frames).

Acknowledgments The authors want to acknowledge the Natural Sciences and Engineering Research Council of Canada (NSERC) and the Canadian Institutes of Health Research (CIHR) for funding the acquisition of the validation images.

\section{Compliance with ethical standards}

Conflict of interest The authors declare that they have no conflict of interest.

\section{Appendix A: statistical shape model}

A SSM is a set of shapes on which the location of the landmarks is correspondent. From this set of shapes, we can extract the mean model $\overline{\mathbf{M}}$ and the covariance matrix, from which we can compute the eigenvectors (modes of variation) and the eigenvalues.

$\mathbf{D}=\frac{1}{K-1} \sum_{k=1}^{K}\left(\mathbf{M}_{k}-\overline{\mathbf{M}}\right)\left(\mathbf{M}_{k}-\overline{\mathbf{M}}\right)^{T}$

$\mathbf{D} \cdot \overrightarrow{\mathbf{M}}_{k}=\lambda_{k}^{2} \cdot \overrightarrow{\mathbf{M}}_{k}$

$\sigma_{1}^{2} \geq \lambda_{2}^{2} \geq \cdots \geq \lambda_{K-1}^{2}$

where $K$ is the number of shapes, $\lambda_{k}^{2}$ are the descendingorder eigenvalues of the covariance matrix $\mathbf{D}$, and $\overrightarrow{\mathbf{M}}_{k}$ are the corresponding eigenvectors. To deform a SSM, we can multiply specific weights to the modes and add them to the mean model.

$\mathbf{M}_{S S M}=\overline{\mathbf{M}}+\sum_{k=1}^{K^{\prime}} \beta_{k} \overrightarrow{\mathbf{M}}_{k}$

\section{Appendix B: expectation conditional maximization algorithm for GMMs}

A femur model is represented by a set of 3D points $\mathbf{X}_{s}, s=1, \ldots, S$. A set of fluoroscopic images $I_{j}, j=1, \ldots, J$ are simultaneously acquired with different sources ${ }^{j} \mathbf{S}$ and image planes. On each fluoroscopic image $I_{j}$, the contour of the femur ${ }^{j} \mathbf{y}_{n}$ is semiautomatically segmented. The femur silhouette is defined by points $\mathbf{X}_{m}$, and their projection leads to ${ }^{j} \mathbf{x}_{m}$ where $j$ indicates the image on which the points are projected. We also define a set of virtual observations ${ }^{j} \mathbf{o}_{m}$ that have a correspondent point in the $3 \mathrm{D}$ space $\mathbf{O}_{m}$. The registration problem is the estimation of the homogeneous matrix (expressed by the transformation parameters $\theta$ ) which minimizes the distance between the virtual observation $\mathbf{O}_{m}$ and the silhouette point $\mathbf{X}_{m}$.

The variables used in this description are:

- ${ }^{j} \mathcal{Y}$ is the contour extracted from each image, whose pixels are ${ }^{j} \mathbf{y}_{n}, n=1, \ldots, N$ (also called observations)

- $\mathbf{X}_{s}, s=1, \ldots, S$ are the points of the 3D shape

- ${ }^{j} \mathcal{X}$ is the set of points of the silhouette ${ }^{j} \mathbf{X}_{m}, m=1, \ldots, M<S$

- ${ }^{j} \mathbf{X}_{m}, m=1, \ldots, M$ are the pixel of the shape's silhouette projected on image $j$

- ${ }^{j} \mathbf{o}_{m}, m=1, \ldots, M$ are the virtual observations on the image $j$

- $\mathbf{O}_{m}, m=1, \ldots, M$ are the virtual points backprojected in the 3D space (Fig. 8).

\section{Gaussian model and likelihood}

Each $\mathbf{X}_{s}$ point of the model is defined as the centroid of a 3D Gaussian distribution with mean $\mathbf{X}_{s}$ and covariance 


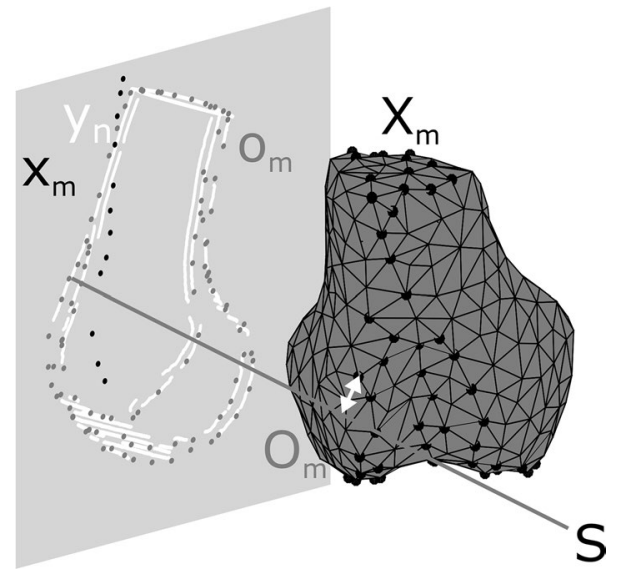

Fig. 8 In the figure are represented the shape with the points $\mathbf{X}_{s}$, the extracted silhouette on the shape $\mathbf{X}_{m}$ and their projection $\mathbf{x}_{m}$ on the image plane. It also represented the source of the X-ray beam $(\mathbf{S})$ and the points extracted from the contour of the image $\mathbf{y}_{n}$, from which we can calculate the virtual observation $\mathbf{o}_{m}$ and its backprojection $\mathbf{O}_{m}$. The white arrow between the backprojected virtual observation $\mathbf{O}_{m}$ and its associated silhouette point $\mathbf{X}_{m}$ is the minimized distance at each iteration

matrix $\Sigma_{s}$, identifying in this way a Gaussian mixture model (GMM). Considering isotropic covariances, each $\Sigma_{s}, s=1, \ldots, S$ is defined as

$\Sigma_{s}=\sigma_{s} \mathbf{I}_{3}$

where $\mathbf{I}_{3}$ is the $3 \times 3$ identity matrix, and $\sigma_{s}$ is the scalar value of the covariance that varies for each $\mathbf{X}_{s}$ point. The operator $\mu: \mathbb{R}^{3} \rightarrow \mathbb{R}^{3}$ transforms a point $\mathbf{X}_{s}$ in another point $\mu\left(\mathbf{X}_{s}, \theta\right)$ where $\theta$ is the parametrization of the transformation.

The likelihood $(\mathcal{L})$ that expresses the probability that the contour is coincident with the silhouette projection is a function of both the registration parameters $\theta$ and the covariances.

$\mathcal{L}\left(\theta, \sigma_{1}, \ldots, \sigma_{S} \mid \mathcal{Y}\right)=\log \mathcal{P}\left(\mathcal{Y} ; \theta, \sigma_{1}, \ldots, \sigma_{S}\right)$

where $\mathcal{P}()$ is the probability that the set of observations $\mathcal{Y}$ is extracted from the GMM with parameters $(\theta, \sigma)$ and the likelihood indicates the probability that the set of observations $\mathcal{Y}$ is coincident with the projection of the shape's silhouette $\mathcal{X}$.

This maximization cannot be performed due to the presence of missing data, as the assignment of each observation to one of the Gaussian of the GMM is unknown. The operator $\left\{Z: \mathbf{y}_{n} \rightarrow \mathbf{x}_{m}\right\}, n=1, \ldots, N$ assigns an observation $\mathbf{y}_{n}$ either to a silhouette model point $\mathbf{x}_{\mathbf{m}}$ or to an outlier class. If $\left(Z: \mathbf{y}_{n} \rightarrow \mathbf{x}_{m}\right)$, then the observation $\mathbf{y}_{n}$ is associated with the point $\mathbf{x}_{m}$, otherwise, if ( $Z: \mathbf{y}_{n} \rightarrow \mathbf{x}_{M+1}$ ), then the observation $\mathbf{y}_{n}$ is an outlier.
The likelihood is replaced by the expected completedata $\log$-likelihood $\mathcal{E}$ conditioned by the observed data, as suggested by Dempster [17].

$\mathcal{E}\left(\theta, \sigma_{1}, \ldots, \sigma_{S} \mid \mathcal{Y}, Z\right)=E_{Z}\left[\log \mathcal{P}\left(\mathcal{Y}, Z ; \theta, \sigma_{1}, \ldots, \sigma_{S}\right) \mid \mathcal{Y}\right]$

To evaluate Eq. (12), the probabilities of the observations must be expressed as a set of probability density functions (PDFs). $p_{m}=\mathcal{P}\left(Z: \mathbf{y}_{n} \rightarrow \mathbf{x}_{m}\right)$ is the prior probability that the observation $\mathbf{y}_{n}$ belongs to the cluster $m$ with center $\mu\left(\mathbf{x}_{m} ; \theta\right)$ while $p_{M+1}=\mathcal{P}\left(Z: \mathbf{y}_{n} \rightarrow \mathbf{x}_{M+1}\right)$ expresses the prior probability of $\mathbf{y}_{n}$ to be an outlier.

$p_{m}= \begin{cases}\mathcal{P}\left(Z: \mathbf{y}_{n} \rightarrow \mathbf{x}_{m}\right)=\frac{a}{A} & \text { if } 1 \leq m \leq M \\ \mathcal{P}\left(Z: \mathbf{y}_{n} \rightarrow \mathbf{x}_{M+1}\right)=\frac{A-M a}{A} & \text { if } m=M+1\end{cases}$

In Eq. (13), the variable $a$ indicates a small circular area $\left(a=\pi r^{2}\right)$ around the center of the projected GMM $\mu\left(\mathbf{x}_{m}, \theta\right)$, whereas $A$ indicates the whole volume of work, so that $a \ll A$. The likelihood of an observation $\mathbf{y}_{n}$ given its assignment to cluster $m$ is drawn from a normal distribution:

$\mathcal{P}\left(\mathbf{y}_{n} \mid Z: \mathbf{y}_{n} \rightarrow \mathbf{x}_{m}\right)=\mathcal{N}\left(\mathbf{y}_{n} \mid \mu\left(\mathbf{x}_{m} ; \theta\right), \sigma_{m}\right)=\frac{1}{\sigma_{m} \sqrt{2 \pi}} e^{-\frac{\left\|\mathbf{y}_{n}-\mathbf{x}_{m}\right\|^{2}}{2 \sigma_{m}^{2}}}$

and the same likelihood of the observation given its assignment to the outlier class is a uniform distribution over the area $A$

$\mathcal{P}\left(\mathbf{y}_{n} \mid Z: \mathbf{y}_{n} \rightarrow \mathbf{x}_{M+1}\right)=\mathcal{U}\left(\mathbf{y}_{n} \mid A, 0\right)=\frac{1}{A}$

The marginal distribution of an observation is:

$\mathcal{P}\left(\mathbf{y}_{n}\right)=\sum_{m=1}^{M+1} p_{m} \mathcal{P}\left(\mathbf{y}_{n} \mid Z: \mathbf{y}_{n} \rightarrow \mathbf{x}_{m}\right)$

Equation (11) then becomes

$\log \mathcal{P}(\mathcal{Y})=\sum_{m=1}^{M} \log \left(\sum_{n=1}^{N} p_{n} \mathcal{N}\left(\mathbf{y}_{m} \mid \mu\left(\mathbf{x}_{n} ; \theta\right), \sigma_{n}\right)+\frac{p_{n+1}}{A}\right)$,

and Eq. (12) becomes

$$
\begin{aligned}
& \mathcal{E}\left(\theta, \sigma_{1}, \ldots, \sigma_{S} \mid \mathcal{Y}, \mathcal{Z}\right) \\
& \quad=\sum_{\mathcal{Z}} \mathcal{P}\left(\mathcal{Z} \mid \mathcal{Y}, \theta, \sigma_{1}, \ldots, \sigma_{S}\right) \log \mathcal{P}\left(\mathcal{Y}, \mathcal{Z} ; \theta, \sigma_{1}, \ldots, \sigma_{S}\right)
\end{aligned}
$$

\section{Expectation Maximization}

The expectation conditional maximization method is an iterative way to solve the maximum likelihood problem of 
Eq. (12). Starting from an initial estimate of the parameters, the method computes the posterior probabilities given the current parameters and covariances and then maximizes the expectation in (12) with respect to the registration parameters (given the current covariances) and the covariances (given the newly estimated parameters).

\section{Expectation step}

The expectation step is defined as the computation of the posterior probabilities given the current estimate of the registration parameters and the covariance matrix. In this case, the posterior probability is computed between the contour points $\left({ }^{j} \mathbf{y}_{n}\right)$ and the projection of the silhouette on the 2D images $\left({ }^{j} \mathbf{x}_{m}\right)$. Recovering the Eqs. (13), (14), (15), and (16) and using the Bayes' rule, the expression for the posterior probability becomes:

$$
\begin{aligned}
p_{m n}^{q}= & \mathcal{P}\left(Z: \mathbf{y}_{n} \rightarrow \mathbf{x}_{m} \mid \mathbf{y}_{n} ; \theta^{q}, \sigma^{q}\right) \\
= & \frac{\mathcal{P}\left(\mathbf{y}_{n} \mid Z: \mathbf{y}_{n} \rightarrow \mathbf{x}_{m}\right) \mathcal{P}\left(Z: \mathbf{y}_{n} \rightarrow \mathbf{x}_{m}\right)}{\mathcal{P}\left(\mathbf{y}_{n}\right)} \\
= & \frac{\sigma_{m}^{-2} e^{\frac{-\left\|\mathbf{y}_{n}-\mathbf{x}_{m}\right\|^{2}}{2 \sigma_{m}^{2}}}}{\sum_{i=1}^{M} \sigma_{i}^{-2} e^{\frac{-\left\|\mathbf{y}_{n}-\mathbf{x}_{i}\right\|^{2}}{2 \sigma_{i}^{2}}}+c}
\end{aligned}
$$

with $c$ that is the outlier component:

$c=2 r^{-2}$

\section{Conditional maximization step}

The conditional maximization step aims at maximizing the likelihood described in Eqs. (11) and (12). It uses the definition of virtual observation, that is, a normalized sum over all the observations weighted by their posterior probability [21]. The virtual observation $O$ and its weight $\lambda$ are obtained for each model point $x_{n}$ using the posterior probabilities $p_{m n}^{q}$ and the observations $y_{m}$ :

$$
\begin{aligned}
v_{n} & =\sum_{m=1}^{M} p_{m n} \\
\mathbf{o}_{n} & =\frac{1}{v_{n}} \sum_{m=1}^{M} p_{m n} \mathbf{y}_{m}
\end{aligned}
$$

Equation (12) can be rewritten replacing the conditional probabilities with the normal and uniform distribution as expressed in Eq. (22) (for the complete steps, the reader can refer to [21])

$\mathcal{E}=-\frac{1}{2} \sum_{m=1}^{M} \sum_{n=1}^{N} \frac{p_{m n}}{\sigma_{n}^{2}}\left(\left\|\mathbf{y}_{m}-\mu\left(\mathbf{X}_{n}, \theta\right)\right\|^{2}+\log \left(\sigma_{n}^{2}\right)\right)$
The minimization of Eq. (22) over $\theta$ keeping constant the covariances $\sigma$ leads to:

$\theta^{q+1}=\arg \min _{\theta} \frac{p_{m n}}{\sigma_{n}^{2}}\left\|\mathbf{y}_{m}-\mu\left(\mathbf{X}_{n}, \theta\right)\right\|^{2}+\frac{\rho}{2}\|L(\mu)\|^{2}$

where $\|L(\mu)\|^{2}$ is a regularization term over the parameters. Equation (23) can be simplified using the definitions of Eq. (21):

$\theta^{q+1}=\arg \min _{\theta} v_{n}\left\|\mathbf{O}_{n}-\mu\left(\mathbf{X}_{n}, \theta\right)\right\|^{2}+\frac{\rho}{2}\|L(\mu)\|^{2}$

where $\mathbf{O}_{n}$ is the 3D point nearest to $\mathbf{X}_{n}$ on the ray backprojected from $\mathbf{o}_{n}$. A 2D/3D registration problem is now cast into a $3 \mathrm{D} / 3 \mathrm{D}$ registration that can be solved using already addressed solutions [4, 10, 21, 37].

The second step of the conditional maximization is the update of the covariances, using the registration parameters newly computed:

$\sigma_{n}^{2}=\frac{\sum_{m=1}^{M} p_{m n}|| \mathbf{y}_{m}-\mu\left(\mathbf{x}_{n}, \theta\right) \|^{2}}{2 \sum_{m=1}^{M} p_{m n}}$

In Eq. (25), the value $\mu\left(\mathbf{x}_{n}, \theta\right)$ is the projection of the $3 \mathrm{D}$ point $\mathbf{X}_{n}$ updated with the parameters $\theta$.

\section{References}

1. Acker S, Li R, Murray H, John P, Banks S, Mu S, Wyss U, Deluzio K (2011) Accuracy of single-plane fluoroscopy in determining relative position and orientation of total knee replacement components. J Biomech 44(4):784-787

2. Akbari Shandiz M (2015) Component placement in hip and knee replacement surgery: device development, imaging and biomechanics. $\mathrm{PhD}$ thesis, Biomedical Engineering

3. Anderst W, Zauel R, Bishop J, Demps E, Tashman S (2009) Validation of three-dimensional model-based tibio-femoral tracking during running. Med Eng Phys 31(1):10-16

4. Arun KS, Huang TS, Blostein SD (1987) Least-squares fitting of two 3-d point sets. Pattern Anal Mach Intell IEEE Trans (5):698-700

5. Baka N, Kaptein B, de Bruijne M, van Walsum T, Giphart J, Niessen WJ, Lelieveldt BP (2011) 2d-3d shape reconstruction of the distal femur from stereo $\mathrm{X}$-ray imaging using statistical shape models. Med Image Anal 15(6):840-850

6. Baka N, de Bruijne M, van Walsum T, Kaptein B, Giphart J, Schaap M, Niessen WJ, Lelieveldt BP (2012) Statistical shape model-based femur kinematics from biplane fluoroscopy. Med Imaging IEEE Trans 31(8):1573-1583

7. Baka N, Kaptein B, Giphart J, Staring M, de Bruijne M, Lelieveldt B, Valstar E (2014) Evaluation of automated statistical shape model based knee kinematics from biplane fluoroscopy. $\mathbf{J}$ Biomech 47(1):122-129

8. Beretta E, De Momi E, Camomilla V, Cereatti A, Cappozzo A, Ferrigno G (2014) Hip joint centre position estimation using a dual unscented kalman filter for computer-assisted orthopaedic surgery. Proc Inst Mech Eng H J Eng Med 228(9):971-982 
9. Beretta E, Valenti M, De Momi E, Ferrigno G (2012) Hip joint center location with kalman filter. J Bone Joint Surg, British Volume, 94-B(SUPP XLIV):68

10. Besl PJ, McKay ND (1992) Method for registration of 3-d shapes. In: Robotics-DL tentative, pp 586-606, International society for optics and photonics

11. Bijlsma J, Knahr K (2007) Strategies for the prevention and management of osteoarthritis of the hip and knee. Best Pract Res Clin Rheumatol 21(1):59-76

12. Canny J (1986) A computational approach to edge detection. Pattern Anal Mach Intell IEEE Trans 6:679-698

13. Chui H, Rangarajan A (2003) A new point matching algorithm for non-rigid registration. Comput Vis Image Underst 89(2):114-141

14. Cootes TF, Cooper DH, Taylor CJ, Graham J (1991) A trainable method of parametric shape description. In BMVC91, pp 54-61, Springer

15. Costa Filho IG (2008) Mixture models for the analysis of gene expression: integration of multiple experiments and cluster validation. $\mathrm{PhD}$ thesis, Freie Universität Berlin

16. De Momi E, Beretta E, Ferrigno G (2013) Hip joint centre localisation with an unscented kalman filter. Comput Methods Biomech Biomed Eng 16(12):1319-1329

17. Dempster AP, Laird NM, Rubin DB (1977) Maximum likelihood from incomplete data via the em algorithm. J R Stat Soc. Series B (methodological), 1-38

18. Dennis D, Mahfouz M, Komistek R, Hoff W (2005) In vivo determination of normal and anterior cruciate ligament-deficient knee kinematics. J Biomech 38:241-53

19. Ferrigno G, Pedotti A (1995) Opto-electronics based systems. In: Allard P, Stokes IA, Bianchi JP (eds) Three-dimensional analysis of human movement, human kinetics 1st ed, pp 57-78, Human Kinetics Publishers

20. Ho K, Saevarsson S, Ramm H, Lieck R, Zachow S, Sharma G, Rex E, Amiri S, Wu B, Leumann A et al (2012) Computed tomography analysis of knee pose and geometry before and after total knee arthroplasty. J Biomech 45(13):2215-2221

21. Horaud R, Forbes F, Yguel M, Dewaele G, Zhang J (2011) Rigid and articulated point registration with expectation conditional maximization. Pattern Anal Mach Intell IEEE Trans 33(3):587-602

22. Juneja M, Sandhu PS (2009) Performance evaluation of edge detection techniques for images in spatial domain. Int J Comput Theory Eng 1(5):614-621

23. Kittler J (1983) On the accuracy of the sobel edge detector. Image Vis Comput 1(1):37-42

24. Li J-S, Tsai T-Y, Wang S, Li P, Kwon Y-M, Freiberg A, Rubash HE, Li G (2014) Prediction of in vivo knee joint kinematics using a combined dual fluoroscopy imaging and statistical shape modeling technique. J Biomech Eng 136(12):124503

25. Litwic A, Edwards MH, Dennison EM, Cooper C (2013) Epidemiology and burden of osteoarthritis. British Medical Bulletin, $\mathrm{p}$ $1 \mathrm{ds} 038$

26. Lorensen W, Cline H (1987) Marching cubes: A high resolution 3d surface construction algorithm. In: ACM Siggraph Computer Graphics, vol 21, pp 163-169, ACM

27. Metz C (2005) Digitally reconstructed radiographs. Utrecht: Utrecht University, p 79

28. Miranda D, Rainbow M, Leventhal E, Crisco J, Fleming B (2010) Automatic determination of anatomical coordinate systems for three-dimensional bone models of the isolated human knee. J Biomech 43(8):1623-1626
29. Sharma G, Saevarsson S, Amiri S, Montgomery S, Ramm H, Lichti D, Lieck R, Zachow S, Anglin C (2012) Radiological method for measuring patellofemoral tracking and tibiofemoral kinematics before and after total knee replacement. Bone Joint Res 1(10):263-271

30. Tashman S, Anderst W (2003) In-vivo measurement of dynamic joint motion using high speed biplane radiography and CT: Application to canine ACL deficiency. J Biomech Eng 125(2):238

31. Tersi L, Barré A, Fantozzi S, Stagni R (2013) In vitro quantification of the performance of model-based mono-planar and biplanar fluoroscopy for $3 \mathrm{~d}$ joint kinematics estimation. Med Biol Eng Comput 51(3):257-265

32. Tsai T-Y, Lu T-W, Chen C-M, Kuo M-Y, Hsu H-C (2010) A volumetric model-based $2 \mathrm{~d}$ to $3 \mathrm{~d}$ registration method for measuring kinematics of natural knees with single-plane fluoroscopy. Med Phys 37(3):1273-1284

33. Valenti M, De Momi E, Yu W, Ferrigno G, Zheng G (2014) $2 \mathrm{~d} / 3 \mathrm{~d}$ ssm reconstruction method based on robust point matching. Bone Joint J Orthop Proc Suppl 96(SUPP 16):1-1

34. Valenti M, De Momi E, Yu W, Ferrigno G, Akbari Shandiz M, Anglin C, Zheng G (2015) Fluoroscopy-based tracking of femoral kinematics with statistical shape models. Int J Comput Assist Radiol Surgery, 1-9

35. Yu W, Zheng G (2014) Personalized x-ray reconstruction of the proximal femur via a new control point-based $2 \mathrm{~d}-3 \mathrm{~d}$ registration and residual complexity minimization. In: Eurographics workshop on visual computing for biology and medicine, VCBM 2014, Vienna, Austria, 2014. Proceedings, pp 155-162

36. Zhang W, Moskowitz R, Nuki G, Abramson S, Altman R, Arden N, Bierma-Zeinstra S, Brandt K, Croft P, Doherty M et al (2007) OARSI recommendations for the management of hip and knee osteoarthritis, part i: critical appraisal of existing treatment guidelines and systematic review of current research evidence. Osteoarthritis Cartilage 15(9):981-1000

37. Zheng G (2013) Expectation conditional maximization-based deformable shape registration. In: Computer analysis of images and patterns, pp 548-555, Springer

38. Zheng G, Dong X, Rajamani K, Zhang X, Styner M, Thoranaghatte R, Nolte L, Ballester M (2007) Accurate and robust reconstruction of a surface model of the proximal femur from sparsepoint data and a dense-point distribution model for surgical navigation. IEEE Trans Bio-Med Eng 54:2109-22

39. Zhu Z, Li G (2012) An automatic 2d-3d image matching method for reproducing spatial knee joint positions using single or dual fluoroscopic images. Comput Methods Biomech Biomed Eng 15(11):1245-1256

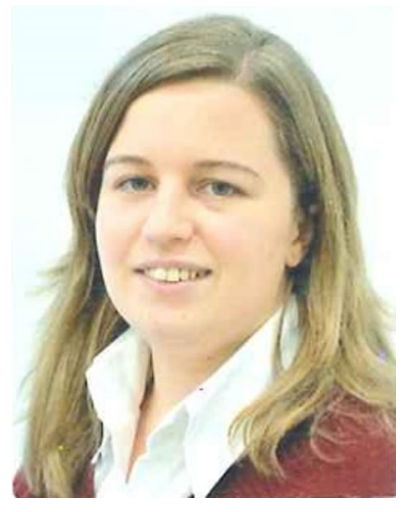

Marta Valenti received the M.Sc. degree in Biomedical Engineering from Politecnico di Milano in 2012. She obtained the Ph.D. in Bioengineering on Surgery Planning in Orthopedics. 

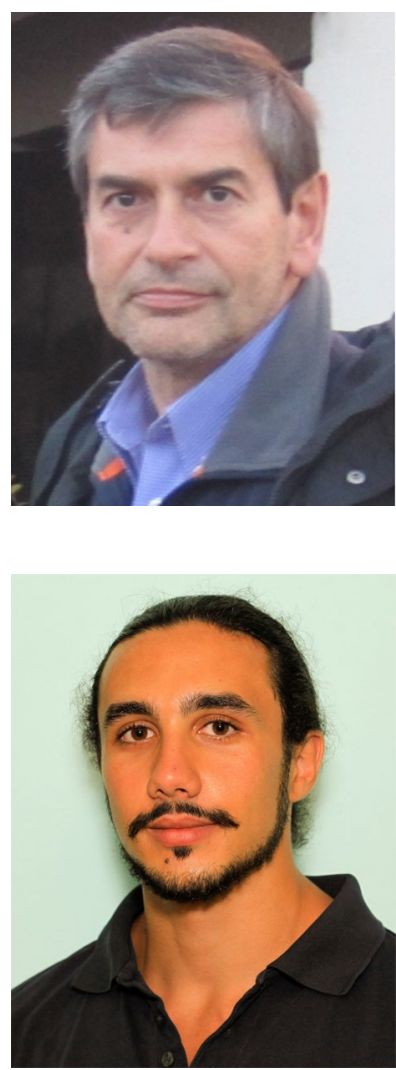

Dario Martina is currently pursuing the bachelor's degree in Biomedical Engineering at Politecnico di Milano, with a thesis on $2 \mathrm{D} / 3 \mathrm{D}$ registration method comparison.

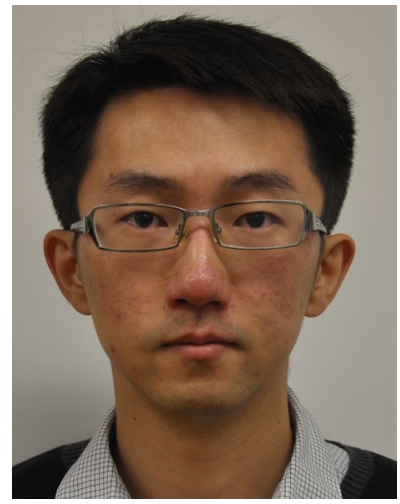

Weimin Yu received the M.Sc. in BME from Southeast University, China, in 2008. He is pursuing the Ph.D. degree with University of Bern, where he is doing research on the medical image analysis.

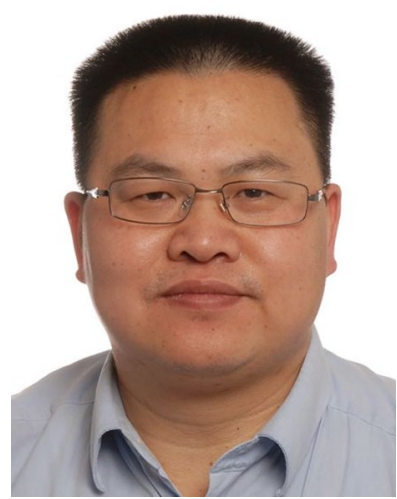

Guoyan Zheng received his Ph.D. degree in BioEngineering from University of Bern in 2002. He is Associate Professor and Head of the Information Processing in Medical Interventions (IPMI) group

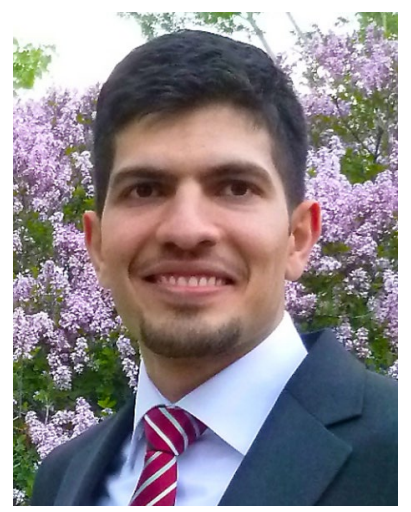

Mohsen Akbari Shandiz completed his Ph.D. degree in Biomedical Engineering at University of Calgary. His current research interests include medical imaging, biomechanics, and computer-assisted surgery.

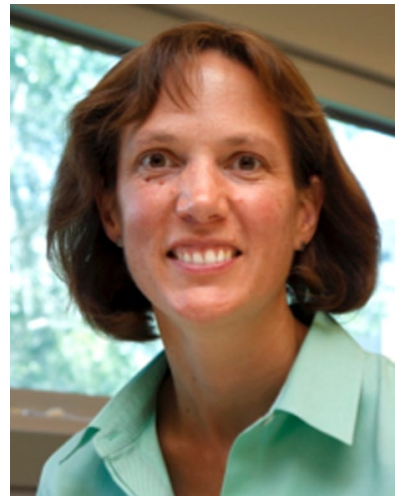

Carolyn Anglin is an Associate Professor in Biomedical Engineering at the University of Calgary. Her fields of research include surgical training models, biomechanics, and medical imaging.

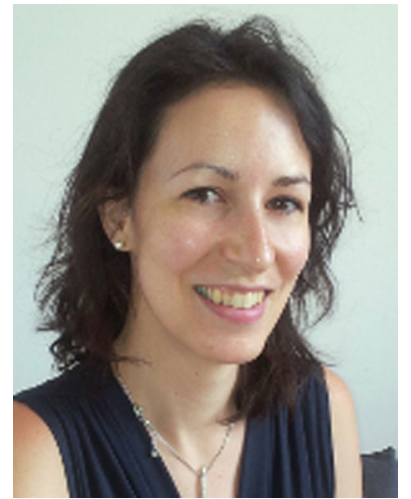

Elena De Momi completed her Ph.D. in Bioengineering (2006), M.Sc. in Biomedical Engineering (2002), and is a Assistant Professor at Department of Electronics, Information and Bioengineering at Politecnico di Milano. 